\title{
Preparedness to face the COVID-19 pandemic - is India missing the mark?
}

\author{
Devasahayam J Christopher, Barney TJ Isaac, Balamugesh Thangakunam
}

Department of Pulmonary Medicine, Christian Medical College, Vellore, India

\begin{abstract}
$\mathrm{T}$
\end{abstract} he Corona Virus Disease (COVID-19) caused by SARS Co-V 2 in late 2019 has resulted in a pandemic infecting more than 8 million across the world with nearly half a million lives already lost. In Wuhan, the epicentre of the disease in China and in Singapore, after an apparent suppression of the pandemic, there has been a rebound of cases. India, despite being on lock down for more than two months witnessed a rise in cases, and this is likely to dramatically worsen as lock down has been released. As part of the preparation for the management of COVID-19 cases, efforts are in full swing and the need to augment the invasive mechanical ventilation (IMV) capacity seems to be a part of the strategy. It is clear that only around $5 \%$ of those infected will require critical care and a proportion of them may end up on IMV. More importantly, the outcome in those ventilated is poor- $60 \%$ mortality in the best case scenario and as bad as $97 \%$ in the worst. IMV is expensive and requires

The cost of procuring invasive mechanical ventilation is high and outcomes of ventilated COVID-19 patients are poor and hence it may not be a cost effective investment and may deplete the scarce resources in resource limited settings. skilled manpower, which cannot be created in a short span of time. On the other hand, 14\% of the COVID-19 patients may require admission and only good supportive care, primarily oxygen therapy and non-invasive ventilation. Appropriate preparation to provide good supportive care is crucial. This could avert or reduce the need for IMV and is likely to save more lives. Such a model of cost-effective care is more appropriate for India and this may be something that other resource limited countries could emulate.

\section{REBOUND OF CASES}

According to John Hopkin's Corona virus resource centre the number of confirmed cases with COVID-19 was 8079076 with 438171 deaths as of 16th June 2020 [1]. A variety of measures are being taken by countries around the world to slow the spread of the disease. As of 21st April 2020 more than a third of the planet's population is under some form of restriction [2]. However the cases and deaths are continuing to rise in most countries despite the lock down. Mathematical modelling has predicted that sustained lockdown or repeated lockdown is going to be required to prevent rebound of infections [3]. The lockdown in India which commenced on 24th March 2020, had been effective in blunting the exponential growth to a linear growth. After a few rounds of extensions, the lockdown was released by the end of May in a phased manner. This has already resulted in a rebound of cases and lockdowns are again being considered in selected hotspots of the country. 


\section{THE HEALTH CARE NEEDS AND CAPACITY}

Based on the current trend, a team of modelling experts convened under the leadership of the principal scientific advisor to the government of India have modelled the likely scenario weeks down the line, using a statistical model 'COVID-19 Med Inventory [4]. The prediction is that by mid-July, there could be a steep increase in the cases in India to 30,49 000 with 79000 requiring ICU beds, 55000 requiring ventilators and 5700 dying every day. This situation is likely to worsen further.

The Indian health ministry has revealed [5] that there are 15980 isolation beds and 37326 quarantine beds for the care of COVID-19 patients. The Indian Society of Critical Care Medicine, estimates that there could at best be 100000 ICU beds and between 35000 and 45000 ventilators in the country (mostly in the private sector, and out of reach of the vast majority of the population) [6]. Since these beds would be required to manage a variety of critically ill medical and surgical patients, it is not clear how much could be set apart for the care of COVID-19 patients.

An empowered committee of the ministry of health has asked the senior industry leaders to amplify the logistics supply chain for ventilators and PPEs. They have appealed to both the public and private sector [7]. Adding to the shortage of equipment, skilled workforce is required to manage these patients, and India would fall woefully short [6].

\section{OUTCOMES WITH INVASIVE MECHANICAL VENTILATORY SUPPORT}

As India prepares to address the imminent escalation of health care needs from this pandemic, the aim would be to save maximum number of lives. It is important to understand the cost-benefit ratios of the various interventions, in order to make optimal use of the limited resources. In nearly $81 \%$ of symptomatic patients, the disease is mild, and these individuals are likely to get better without any specific measures [8]. Around 14\% may have moderate symptoms and need hospitalisation due to hypoxia but not intensive care unit (ICU) care and an additional 5\% may require care in an ICU, due to multiorgan failure or refractory hypoxia [8]. The need for invasive mechanical ventilation (IMV) in those who are critically ill is high ranging from 42 to 100 percent in various published cohorts [9].

On the other hand, setting up medical wards with adequate facilities for good supportive care and oxygen treatment and whenever possible, providing non-invasive ventilation services may result in lot of lives saved.
The outcome of patients who needed invasive ventilation has been abysmal. In the initial report from 552 hospitals across China[10], the mortality rate of those on IMV was $60 \%$ (assuming all who died in hospital were ventilated); this is despite one fifth of them having received ECMO. In a subsequent report from China, the mortality among those on IMV was 97\% [11]. A large cohort [12] of 5700 hospitalised patients from New York City area reported $88.1 \%$ mortality among those who received IMV.

\section{GOOD SUPPORTIVE CARE IS THE WAY FORWARD}

Although several large randomized drug trials are under way, current survival from severe COVID-19 depends entirely on providing the best possible supportive care [13]. It is amply clear that only a small proportion of patients require invasive ventilation, who despite this treatment have poor outcomes. Invasive ventilation is resource intensive and requires a highly skilled team, which cannot be created at short notice. Therefore, in resource limited settings, it would be more fruitful and many more lives may be saved by focussing on good supportive care, which would result in less patients needing IMV. All health care providers who monitor asymptomatic/mildly symptomatic patients or the patients themselves should use a pulse oximeter, which is economical and easily available to assess desaturation and thus decrease the need for hospitalisation. As a rule of thumb, adults with room air oxygen saturation $\leq 93 \%$ [8] could be con-sidered for hospitalisation.

Providing supplemental oxygen is central to the care of hospitalised patients. In 2015, the Lancet Commission on Global Surgery revealed that approximately one-quarter of hospitals surveyed in resourcelimited countries lack sufficient oxygen supply [14]. Thus hospitals being prepared to care for COVID-19 patients requiring hospital admission should identify sources for procuring high-quality medical grade oxygen. Common oxygen sources are oxygen generating plants, liquid oxygen storage tanks, oxygen cyl- 


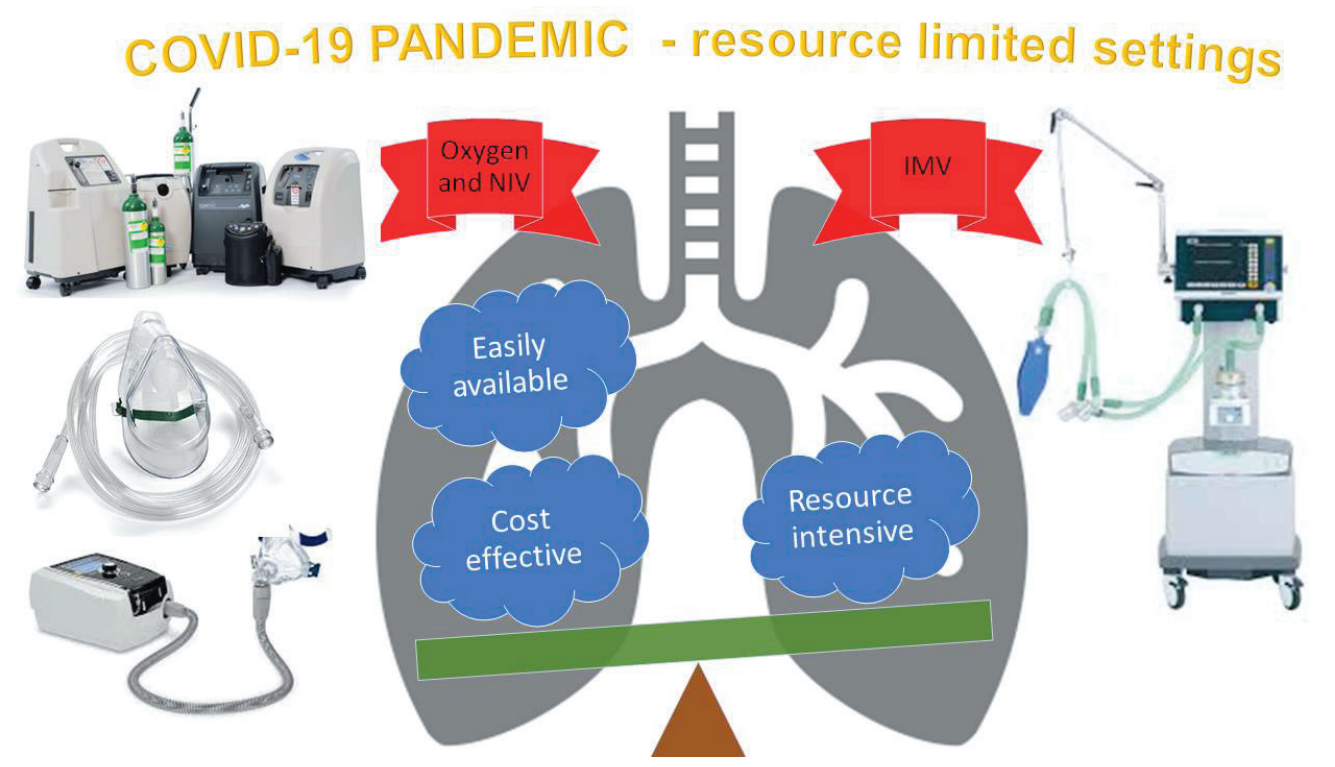

Photo: From the authors' own collection, used with permission.

inder and oxygen concentrator. The total oxygen requirement for a hospital can be calculated using the WHO essential resource planning tool. There should be a well-defined plan to boost oxygen delivery capacity in case there is a surge of patients with COVID-19 requiring oxygenation [15].

Oxygen cylinders are the most frequently used mode and these are purchased by small hospitals and clinics for their requirements. They are bulky and transporting cylinders for refilling is labour intensive. Bed side oxygen concentrators are very helpful plug-and-use devices which can be easily operated with minimum training. Most of the current concentrators can deliver up to 6 lit/min oxygen flow and may be enough for moderately hypoxic patients with COVID-19. In case higher flows are required multiple concentrators can be serially connected. Effort should be taken to scale up the availability of concentrators. They are more important and useful for a greater number of patients than invasive ventilators and far less expensive.

In resource limited settings where invasive ventilators cannot be used (because they are not available, or expertise is not available) simple and less costly equipment which are easier to use (like non-invasive ventialtion (NIV) or CPAP) can be used [13]. Continuous positive airway pressure (CPAP) is now being advocated by some physicians who are managing COVID-19 patients. It could prevent or delay the need for invasive ventilation. CPAP is commonly used in the treatment of obstructive sleep apnea and these devices are available at a modest cost. This can be combined with additional oxygen, either entrained to the device or given through small bore nasal prongs. It is relatively simple to use and it is not difficult to teach physicians caring for COVID-19 patients the operation of this machine. However, to prevent dispersion of exhaled air a viral filter needs to be introduced in the circuit. Prone ventilation has been advocated in the management of patients not only on IMV but also on NIV/CPAP or just on oxygen supplementation if they do not have any contraindication [16]. This does not involve much cost and could facilitate the oxygenation of these patients by reducing the VQ mismatch, recruitment of posterior lung segments, decrease shunting and augmenting secretion clearance [16]. Use of CPAP with prone positioning may be a good strategy to defer or avoid IMV.

\section{CONCLUSION}

In preparation for the imminent escalating health care needs of the COVID-19 pandemic, various strategies are being put in place. Augmentation of bed strength and apportioning beds specifically for care of COVID-19 patients is crucial and a challenge India may struggle to achieve if the predicted increase of cases becomes a reality, on account of poor public health care system and resource constraints. Undue emphasis has been placed on procurement of additional ventilators, which would deplete the available resources and provide very little additional returns. We feel prompt identification of hypoxia by careful monitoring, providing good supportive care for hospitalised patients with infrastructure to provide supplemental oxygen and optimal use of Non-invasive ventilatory modes to defer or delay IMV, will save more lives. This is a model India can create and pass on to other resource-limited countries. 
Funding: None:

Authorship contributions: DJC contributed to the concept, literature review, writing of manuscript, critical revision and final approval of the manuscript. BT contributed by literature review, writing of manuscript, critical revision and final approval of the manuscript. BTJI is the corresponding author and contributed by literature review, writing of manuscript, critical revision and final approval of the manuscript.

Competing interests: The authors completed the ICMJE Unified Competing Interest form (available upon request from the corresponding author), and declare no conflicts of interest.

1 COVID-19 Map. Johns Hopkins Coronavirus Resource Center. Available: https://coronavirus.jhu.edu/map.html. Accessed: 16 June 2020.

2 Denmark enacted a lockdown on March 11. It has now reopened some schools. Business Insider. Available: https://www. businessinsider.in/international/news/a-third-of-the-global-population-is-on-coronavirus-lockdown-x2014hereaposs-our-constantly-updated-list-of-countries-and-restrictions/slidelist/75208623.cms. Accessed: 21 April 2020.

3 Singh R, Adhikari R. Age-structured impact of social distancing on the COVID-19 epidemic in India. ArXiv 2020: ArXiv200312055 Cond-Mat Q-Bio. Availabe: https://arxiv.org/abs/2003.12055. Accessed: 21 April 2020.

4 COVID-19: Medical Resources and Inventory Projection System. Available: https://covid19medinventory.in. Accessed: 16 June 2020.

5 Health ministry. 15,980 isolation beds, 37,326 quarantine beds. The Indian Express. 2020. Available: https://indianexpress.com/article/india/coronavirus-health-ministry-15980-isolation-beds-37326-quarantine-beds-6321114/. Accessed: 23 April 2020

6 Christopher DJ, Isaac BT, Rupali P, Thangakunam B. Health-care preparedness and health-care worker protection in COVID-19 pandemic. Lung India. 2020;37:238-45. Medline:32367846 doi:10.4103/lungindia.lungindia_189_20

7 Covid-19: 'Make in India' push for ventilators, PPEs. India News - Times of India. Available: https://timesofindia.indiatimes.com/india/covid-19-make-in-india-push-for-ventilators-ppes/articleshow/74988501.cms?utm_ source=contentofinterest\&utm_medium=text\&utm_campaign=cppst. Accessed: 23 April 2020.

$8 \mathrm{Wu}$ Z, McGoogan JM. Characteristics of and important lessons from the Coronavirus disease 2019 (COVID-19) outbreak in China: Summary of a report of 72314 cases from the Chinese Center for Disease Control and Prevention. JAMA. 2020;323:1239-42. Medline:32091533 doi:10.1001/jama.2020.2648

9 Need for mechanical ventilation. Available: https:/www.uptodate.com/contents/coronavirus-disease-2019-covid-19-critical-care-issues\#H368577810. Accessed: 24 July 2020.

10 Guan WJ, Ni Z, Hu Y, Liang W, Ou C, He J, et al. Clinical characteristics of Coronavirus disease 2019 in China. N Engl J Med. 2020;382:1708-20. Medline:32109013 doi:10.1056/NEJMoa2002032

11 Zhou F, Yu T, Du R, Fan G, Liu Y, Liu Z, et al. Clinical course and risk factors for mortality of adult inpatients with COVID-19 in Wuhan, China: a retrospective cohort study. Lancet. 2020;395:1054-62. Medline:32171076 doi:10.1016/ S0140-6736(20)30566-3

12 Richardson S, Hirsch JS, Narasimhan M, Crawford JM, McGinn T, Davidson KW, et al. Presenting characteristics, comorbidities, and outcomes among 5700 patients hospitalized with COVID-19 in the New York City area. JAMA. 2020;323:2052-9. Medline:32320003 doi:10.1001/jama.2020.6775

13 Dondorp AM, Hayat M, Aryal D, Beane A, Schultz MJ. Respiratory support in COVID-19 patients, with a focus on resource-limited settings. Am J Trop Med Hyg. 2020;102:1191-7. Medline:32319424 doi:10.4269/ajtmh.20-0283

14 Global Surgery 2030: evidence and solutions for achieving health, welfare, and economic development. Elsevier Enhanced Reader. Available: https://reader.elsevier.com/reader/sd/pii/S014067361560160X?token=335C89DC7C793261 1F895AC7AA79283AE6D80A37B1D60619945727EE4F7E7F2CA1429DB5C42040C94D3C121215BE5C73. Accessed: 2 May 2020.

15 World Health Organization. Oxygen sources and distribution for COVID-19 treatment centres. Available: https://www. who.int/publications-detail/oxygen-sources-and-distribution-for-covid-19-treatment-centres. Accessed: 21 April 2020.

16 Bamford P, Bentley A, Dean J, Whitmore D, Wilson-Baig N. 2020. ICS Guidance for Prone Positioning of the Conscious COVID Patient. 